\title{
Optimal Surface Salinity Perturbations of the Meridional Overturning and Heat Transport in a Global Ocean General Circulation Model
}

\author{
Florian Sévellec, Thierry Huck, Mahdi Ben Jelloul, and Nicolas Grima \\ Laboratoire de Physique des Océans, Université de Bretagne Occidentale, Brest, France \\ JÉRÔME VIALARD \\ IRD, LOCEAN, Paris, France \\ ANTHONY WEAVER \\ CERFACS, Toulouse, France
}

(Manuscript received 9 July 2007, in final form 7 March 2008)

\begin{abstract}
Recent observations and modeling studies have stressed the influence of surface salinity perturbations on the North Atlantic circulation over the past few decades. As a step toward the estimation of the sensitivity of the thermohaline circulation to salinity anomalies, optimal initial surface salinity perturbations are computed and described for a realistic mean state of a global ocean general circulation model [Océan Parallélisé (OPA)]; optimality is defined successively with respect to the meridional overturning circulation intensity and the meridional heat transport maximum. Although the system is asymptotically stable, the nonnormality of the dynamics is able to produce a transient growth through an initial stimulation. Optimal perturbations are calculated subject to three constraints: the perturbation applies to surface salinity; the perturbation conserves the global salt content; and the perturbation is normalized, to remove the degeneracy in the linear maximization problem. Maximization using Lagrangian multipliers leads to explicit solutions (rather than eigenvalue problems), involving the integration of the model adjoint for each value to maximize.

The most efficient transient growth for the intensity of the meridional overturning circulation appears for a delay of $10.5 \mathrm{yr}$ after the perturbation by the optimal surface salinity anomaly. This optimal growth is induced by an initial anomaly located north of $50^{\circ} \mathrm{N}$. In the same way, the most efficient transient growth for the intensity of the meridional heat transport appears for a shorter delay of $2.2 \mathrm{yr}$ after the perturbation by the optimal surface salinity anomaly. This initial optimal perturbation corresponds to a zonal salinity gradient around $24^{\circ} \mathrm{N}$. The optimal surface salinity perturbations studied herein yield upper bounds on the intensity of the response in meridional overturning circulation and meridional heat transport. Using typical amplitudes of the Great Salinity Anomalies, the upper bounds for the associated variability are $0.8 \mathrm{~Sv}$ (1 Sv $\left.\equiv 10^{6} \mathrm{~m}^{3} \mathrm{~s}^{-1}\right)(11 \%$ of the mean circulation) and $0.03 \mathrm{PW}(5 \%$ of the mean circulation), respectively.
\end{abstract}

\section{Introduction}

The ocean circulation is a slow component of the climate system and thus a major contributor to the system's low-frequency variability. Moreover, global warming is likely to influence the oceans' salinity distribution, and hence their dynamics, through the ex-

Corresponding author address: Florian Sévellec, Department of Geology and Geophysics, Yale University, 210 Whitney Ave., P.O. Box 208109, New Haven, CT 06520-8109.

E-mail: florian.sevellec@locean-ipsl.upmc.fr pected modification of the water cycle. Josey and Marsh (2005) have shown that an increase of the precipitation in the North Atlantic subpolar gyre has modified the sea surface salinity since the mid-1970s. Modifications of the salinity field are also found in the deep ocean during recent decades (Curry et al. 2003; Curry and Mauritzen 2005). We propose, therefore, to investigate herein the impact of sea surface salinity perturbations on the ocean circulation.

Oceanic variability is generally associated with either internal or external causes: endogenous variability corresponds to an internal process that sustains the vari- 
ability, while exogenous variability corresponds to an external stimulation that feeds the variability. Nonlinear processes seem to dominate endogenous lowfrequency ocean variability (Dijkstra and Ghil 2005), while linear processes can enhance exogenous variability. The influence of sea surface salinity anomalies on the ocean circulation can thus be studied as an external stimulation of the ocean circulation through freshwater fluxes, as will be done in this paper.

Transient nonnormal growth of initial perturbations can play an important role in atmospheric models (Farrell and Ioannou 1996a), oceanic models (Farrell and Moore 1992; Moore and Farrell 1993; Moore et al. 2002), and coupled models (Moore et al. 2003). Several studies have already addressed the exogenous variability of the thermohaline circulation using this approach.

Tziperman and Ioannou (2002) found optimal perturbations of the thermohaline circulation in a threebox model with respect to the optimal transient growth of the square of the circulation intensity: the rapid decay of the temperature part of the anomaly (through surface restoring) induces a growth of the salinitydominated density anomaly. In their work, though, the response to the optimal stochastic perturbations does not reveal any spectral peak, in spite of the existence of a linear damped oscillatory mode in their model. Zanna and Tziperman (2005) have extended this work by studying optimal initial perturbations in a simplified coupled model, composed of a latitude-depth ocean model with only two levels in the vertical and a onelayer atmosphere. They showed the existence of a transient growth of spatial variance in the transport $40 \mathrm{yr}$ after the perturbation sets in. This growth appears through an increase of both the temperature and salinity perturbations due to the circulation anomalies.

On the other hand, sensitivity studies of the ocean circulation have been made using the adjoint of ocean general circulation models. Sirkes and Tziperman (2001) have found an oscillatory mode with a centennial time scale, by studying the sensitivity of the meridional heat transport (MHT) at $24^{\circ} \mathrm{N}$. In the same way, Bugnion et al. (2006a,b) have studied the sensitivity of the ocean circulation to the forcing terms and have provided sensitivity patterns with respect to the surface heat flux, the surface freshwater flux, or the wind stress.

Sévellec et al. (2007) have reconciled sensitivity and stability studies of the ocean circulation in a latitudedepth model that represents the zonal mean of the thermohaline circulation, with only temperature and salinity as prognostic variables. They obtained impulse, time-invariant, and stochastic optimal forcings of sea surface salinity that influence the thermohaline circulation. Their work also stressed the importance of the circulation function to be maximized and its influence on the results. In addition, such studies allow us to place upper bounds on the response of the meridional overturning circulation (MOC) intensity for a perturbation of a specific amplitude.

As an extension of these previous works, we will address here the exogenous variability in a three-dimensional primitive equation model in a realistic global configuration of $2^{\circ}$ resolution. To better characterize the variability induced by external excitation and determine upper bounds of the response, we will search for the optimal initial perturbation of the sea surface salinity influencing the ocean circulation. A measure of the ocean circulation intensity is thus needed to obtain the optimal perturbation and quantify its impact. We have chosen two different measures: the intensity of the meridional overturning circulation, measuring the baroclinic mass transport of the circulation, and the intensity of the meridional heat transport, measuring the heat transported northward by the circulation. For both measures we are able to find an explicit solution (i.e., not an eigenvalue problem) for the optimal perturbation. The solutions simply involve the propagation of the measure with the adjoint of the ocean model, making these computations numerically feasible to perform even within a realistic global configuration. In addition, for each measure of the circulation, we obtain an upper bound on the modifications induced by the perturbations.

In the first part of this study (section 2) we introduce the nonlinear primitive equation model [Océan Parallélisé (OPA)] and its linearization and adjoint. This low-resolution model is run to a steady state, which is succinctly described. The maximization method is defined, and the computation method fully described. In section 3 , we investigate the optimal initial surface salinity perturbation influencing the meridional overturning circulation: we describe the transient growth of the intensity of the meridional overturning and determine an upper bound on its variability. In section 4 , the same analysis is performed for the intensity of the meridional heat transport. In the last section (section 5), our results are briefly summarized and discussed, and some directions for future work are proposed.

\section{Models and method}

\section{a. The nonlinear model}

In this study, we use the primitive equation ocean model OPA 8.2 (Madec et al. 1998) with the global configuration ORCA2 (Madec and Imbard 1996). This configuration corresponds to an average horizontal 

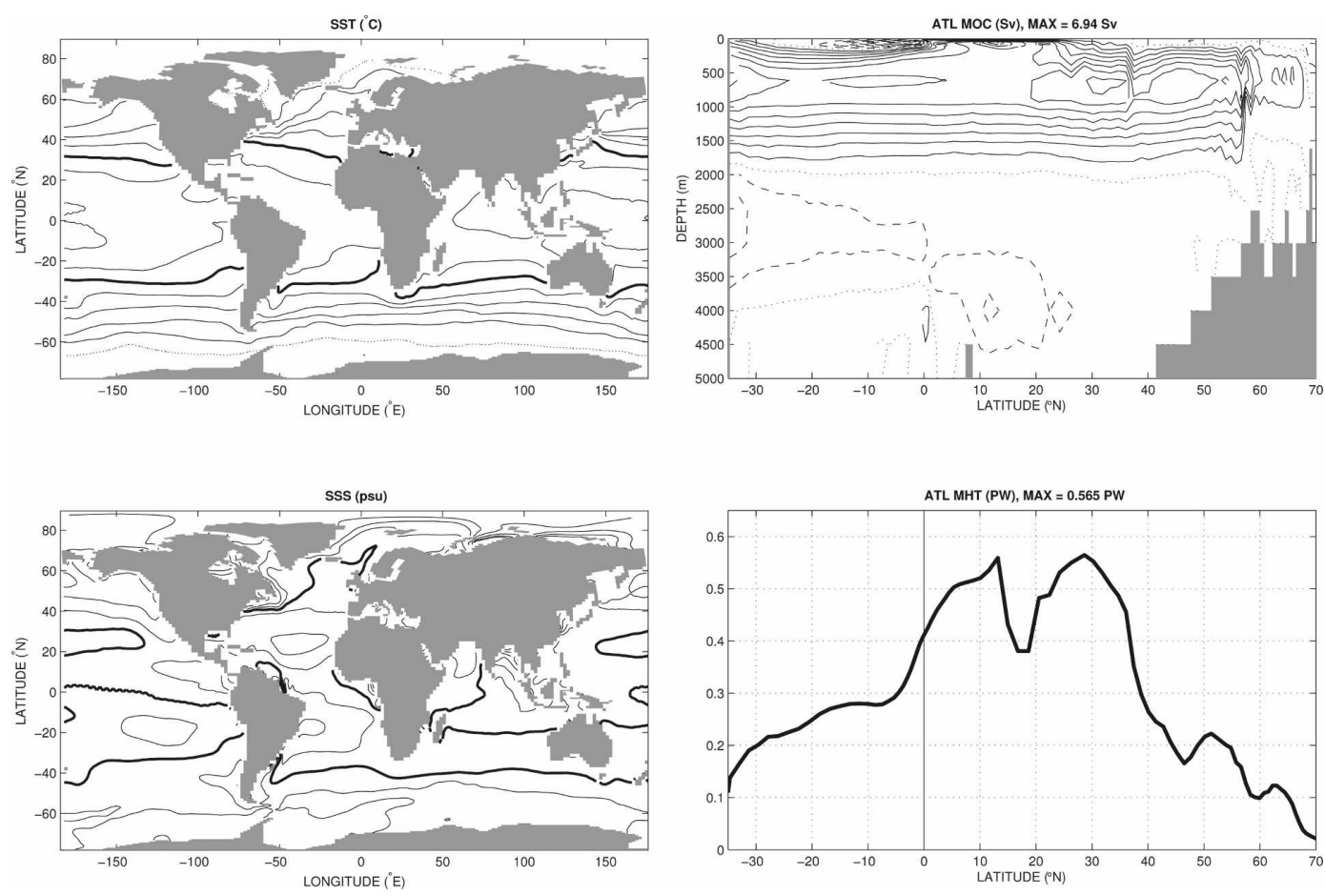

FIG. 1. Steady state of the OPA primitive equation model $2^{\circ}$ global configuration ORCA 2 for a time integration with no seasonal cycle: (top left) SST contoured every $4^{\circ} \mathrm{C}$, with dotted and thick lines corresponding to $0^{\circ}$ and $20^{\circ} \mathrm{C}$; (bottom left) SSS contoured every 1 psu with thick lines corresponding to $35 \mathrm{psu}$; (top right) Atlantic meridional overturning streamfunction, with a maximum value of $7 \mathrm{~Sv}$ at $48^{\circ} \mathrm{N}$ and $600-\mathrm{m}$ depth; (bottom right) Atlantic MHT, with maximum of $0.6 \mathrm{PW}$ at $27^{\circ} \mathrm{N}$. For the MOC, the solid, dashed, and dotted lines correspond to positive, negative, and zeros values; the contours range from -7 to $11 \mathrm{~Sv}$ with an interval of $1 \mathrm{~Sv}$.

resolution of $2^{\circ}$ on 31 vertical levels (whose thickness varies from $10 \mathrm{~m}$ at the surface to $500 \mathrm{~m}$ at depth). The model is integrated on an Arakawa $\mathrm{C}$ grid with $z$ coordinates. We use the model under the rigid-lid approximation that filters the surface gravity waves, but because we are interested in time scales of months to years, this has no impact on our results.

The model configuration uses the following parameterizations: convection is parameterized through an increase of the vertical diffusion when the stratification is unstable; double diffusion takes into account two different terms of mixing for temperature and salinity; the velocities induced by eddies follow Gent and McWilliams (1990); the vertical mixing follows a turbulent closure scheme (Blanke and Delecluse 1993); the mixing coefficient for the horizontal dynamics is a function of longitude, latitude, and depth; and the isopycnal diffusion of temperature and salinity and their mixing coefficients vary in longitude and latitude.

The steady state is obtained by direct time integration of the OPA model under surface boundary conditions that do not vary seasonally. These conditions are obtained by an annual average of all the forcing terms. The surface forcings are the European Centre for Me-
dium-Range Weather Forecasts (ECMWF) fluxes averaged from 1979 to 1993, the European Remote Sensing Satellite (ERS) wind stress [reanalyzed with the Tropical Atmosphere Ocean (TAO) data] averaged from 1993 to 1996, and an annual mean of a seasonal estimation of climatological runoffs. In addition, there is a surface restoring to the Reynolds surface temperature averaged from 1982 to 1989 and to the Levitus climatological salinity (Levitus 1989). Moreover, a mass restoring term on the Levitus climatological values of temperature and salinity is prescribed in the Red and Mediterranean Seas. The time integration of the OPA model, using the Levitus climatological values as initial conditions, shows that the kinetic energy reaches a quasi-steady state after $200 \mathrm{yr}$.

This steady state (Fig. 1) is characterized in the Atlantic Ocean by a northward meridional mass transport above the thermocline and a southward return flow around $1500 \mathrm{~m}$. A recirculation cell exists between 2000 and $4500 \mathrm{~m}$ associated with Antarctic Bottom Water. The MOC intensity in the Atlantic is around $7 \mathrm{~Sv}$ $\left(1 \mathrm{~Sv} \equiv 10^{6} \mathrm{~m}^{3} \mathrm{~s}^{-1}\right)$, as compared to the $18 \mathrm{~Sv}$ from observations (Talley et al. 2003). The discrepancy is mainly due to the removal of the seasonal cycle in the 
surface forcing: this reduces the extrema of the surface temperature, hence the deep-water formation. The associated Atlantic poleward heat transport reaches 0.6 $\mathrm{PW}$ at $27^{\circ} \mathrm{N}$, whereas estimation from inverse calculations and hydrographic sections is $1.3 \mathrm{PW}$ at $24^{\circ} \mathrm{N}$ (Ganachaud and Wunsch 2000). In spite of the weak circulation, the methods employed later are bound to the existence of a steady state; hence, we will proceed with this steady state, keeping in mind that the results might be significantly quantitatively influenced by the weak overturning. Of course, it would be possible to adjust the forcing (using winter values for instance) to obtain a realistic mean state, but this is not the aim of this study. A realistic mean state will be addressed later by considering the full nonautonomous problem of the optimal perturbations on a seasonal cycle (Farrell and Ioannou 1996b).

The nonlinear integration reveals some weak variability (which is poorly resolved in our model) localized in the east equatorial Pacific. However, it has very little impact on the intensity of the Atlantic meridional overturning circulation (less than $10^{-4} \mathrm{~Sv}$ ). To filter these out in our analysis of the optimal perturbation, we compute the steady state as the time average over the last $10 \mathrm{yr}$ of the model integration.

\section{b. The linear and adjoint models}

The models of the linear and adjoint propagator come from the package OPA Tangent Adjoint Model (OPATAM; Weaver et al. 2003). The tangent linear model is a linearization of the primitive equation model described above around a mean state or a trajectory.

We will now prescribe the surface fluxes of heat and freshwater and will not use surface restoring except in the equatorial Pacific (see below). These fluxes are computed as the sum of the prescribed fluxes and the restoring term in the steady state of the nonlinear experiment. This modification remains consistent with the existence of the steady state, but it allows the variability to develop more easily by reducing its damping (Huck and Vallis 2001; Arzel et al. 2006).

Equatorial Pacific variability arises through unstable linear waves, with amplitudes that saturate through nonlinear processes. These must be damped in the linear model integrations focused on the Atlantic Ocean, or the instability will rapidly dominate the global circulation. For this purpose, we apply a restoring term to the steady-state values for the temperature and salinity from $10^{\circ} \mathrm{S}$ to $10^{\circ} \mathrm{N}$ in the Pacific at all depths.

Some approximations have been introduced in the tangent linear and adjoint model. The perturbation is affected by vertical mixing coefficients induced by the mean state, but the coefficient modifications due to the perturbations are neglected. The tracer mixing is acting along the isopycnal surfaces of the mean state, but the variation of these surfaces due to the perturbations is neglected. These approximations imply that in the linear model, there is no feedback of density modifications on diffusion. They should be treated with caution, however, as they may be important when studying the impact of surface salinity modifications on the ocean circulation. Finally, two other linearizations are incomplete: the eddy-induced advection as well as the doublediffusion coefficients are simply computed on the mean state, and their modifications by the perturbations are neglected.

The last simplification in the tangent linear model is the closure of the Mediterranean Sea. An instability occurs at the Strait of Gibraltar in the linear model due to the low resolution of the strait (only one grid point in latitude), which only allows a baroclinic flow. This single horizontal grid point associated with the no-slip lateral boundary condition induces two opposite-sign vortices on each boundary. These two vortices have a positive feedback on the intensity of the zonal velocity through the strait. This mechanism is a plausible explanation of the flow instability there. The closure of the strait wipes out this instability for the linear model integrations. Nevertheless, we still conserve the Mediterranean Sea in the nonlinear model to obtain a better representation of different Atlantic water masses in the steady state. Thus, during the linear and adjoint model integrations, the mean state represents a state with the Mediterranean Sea, and only the perturbations (temperature, salinity, velocities) are absent in the Mediterranean Sea.

\section{c. Maximization method}

The constrained maximization method, using Lagrangian multipliers, has been fully described in Sévellec et al. (2007). Following the same notations, we will develop briefly the solution of the optimal initial perturbation problem. The perturbation state vector is composed of 3D fields of temperature $(T)$, salinity $(S)$, and horizontal velocity (u), and the 2D field of barotropic streamfunction $\left(\psi_{b}\right)$.

We consider a linearization around our steady state leading to the expression of the perturbation state evolution as

$$
|u(t)\rangle=\mathbf{M}(t)|u(0)\rangle,
$$

where $|u(0)\rangle$ is the perturbation state at initial time, $|u(t)\rangle$ is its expression after a time $t$, and $\mathbf{M}(t)$ denotes the propagator from time 0 to $t$. The propagator can be written as

$$
\mathbf{M}(t)=\exp (\mathbf{A} t)
$$


where $\mathbf{A}$ is the Jacobian matrix function of the steady state $|\bar{U}\rangle$.

We use two constraints determined by our physical problem, and a third one to remove the degeneracy of the linear approach. The first one ensures that at initial time the perturbations affect only sea surface salinity (SSS):

$$
|u(0)\rangle=\mathbf{P}\left|u^{\prime}\right\rangle,
$$

where $\left|u^{\prime}\right\rangle$ is the SSS state and $\mathbf{P}$ is the operator reconstructing a full perturbation state with the SSS pattern and setting to zero the rest of the perturbation state. Because we want to investigate the impact of salinity on the ocean circulation, and because salinity perturbations mostly occur at the surface through changes in evaporation, precipitation, ice melting, or runoff, we choose to confine the salinity perturbation to the surface. The second constraint ensures that the initial perturbation conserves salt:

$$
\langle C \mid u(0)\rangle=\iiint d v S=0,
$$

where $\langle C|$ corresponds to the relative volume of each grid point of the salinity field and zero elsewhere. This projection $\langle C \mid u\rangle$ measures the amount of salt of the perturbation $|u\rangle$. Because we focus on a transient growth, our system should return to the same asymptotically stable steady state, hence the total amount of salt should be conserved. The third constraint is required to remove the degeneracy specific to linear maximization problems; it corresponds to a normalization of the initial perturbation:

$$
\langle u(0)|\mathbf{S}| u(0)\rangle-1=0,
$$

where $\mathbf{S}$ is a diagonal matrix defining a quadratic measure of the initial perturbation size. Further calculations show that we only need to define the matrix $\mathbf{N}=\mathbf{P}^{\dagger} \mathbf{S P}$ ( ${ }^{\dagger}$ denotes the adjoint matrix) as follows:

$$
\left\langle u^{\prime}|\mathbf{N}| u^{\prime}\right\rangle=\frac{\iint d s(\mathrm{SSS})^{2} h_{s}}{\iiint d v},
$$

where $h_{s}$ is the surface level thickness. We can define a cost function $G$ that explicitly takes into account the constraints as

$$
\begin{aligned}
G\left(\left|u^{\prime}\right\rangle, \tau\right)= & \left\langle F|\mathbf{M}(\tau) \mathbf{P}| u^{\prime}\right\rangle-\gamma_{1}\left(\left\langle u^{\prime}|\mathbf{N}| u^{\prime}\right\rangle-1\right) \\
& -\gamma_{2}\left\langle C|\mathbf{P}| u^{\prime}\right\rangle,
\end{aligned}
$$

where $\langle F|$ is the bra involved in the unconstrained cost function $\langle F \mid u\rangle ; \gamma_{1}$ and $\gamma_{2}$ are the Lagrange multipliers associated with the constraints (4) and (5), respectively. Differentiating $G$ and solving for the optimal perturbation leads to

$$
\left|u_{0}\right\rangle=\left(2 \gamma_{1}\right)^{-1} \mathbf{P}\left(\mathbf{N}^{-1} \mathbf{P}^{\dagger} \mathbf{M}^{\dagger}(\tau)|F\rangle-\gamma_{2} \mathbf{N}^{-1} \mathbf{P}^{\dagger}|C\rangle\right),
$$

where

$$
\begin{aligned}
\gamma_{1}= & \pm \frac{1}{2}\left[\left\langle F\left|\mathbf{M}(\tau) \mathbf{P N}^{-1} \mathbf{P}^{\dagger} \mathbf{M}^{\dagger}(\tau)\right| F\right\rangle\right. \\
& -\gamma_{2}\left\langle F\left|\mathbf{M}(\tau) \mathbf{P} \mathbf{N}^{-1} \mathbf{P}^{\dagger}\right| C\right\rangle-\gamma_{2}\left\langle C\left|\mathbf{P} \mathbf{N}^{-1} \mathbf{P}^{\dagger} \mathbf{M}^{\dagger}(\tau)\right| F\right\rangle \\
& \left.+\gamma_{2}^{2}\left\langle C\left|\mathbf{P} \mathbf{N}^{-1} \mathbf{P}^{\dagger}\right| C\right\rangle\right]^{1 / 2} \\
\gamma_{2}= & \frac{\left\langle C\left|\mathbf{P} \mathbf{N}^{-1} \mathbf{P}^{\dagger} \mathbf{M}^{\dagger}(\tau)\right| F\right\rangle}{\left\langle C\left|\mathbf{P N} \mathbf{N}^{-1} \mathbf{P}^{\dagger}\right| C\right\rangle} .
\end{aligned}
$$

This explicit solution depends on $\langle F|$ and $\tau$, the time delay after which the transient growth reaches its maximum. Note that $\mathbf{N}^{-1}$ is defined because $\mathbf{S}$ is a diagonal matrix.

\section{Optimal perturbation for the meridional overturning circulation}

We now need to define the cost function $\langle F \mid U\rangle$ appearing in (8) to maximize the intensity of the MOC. Because this intensity corresponds to the streamfunction maximum, which is not a differentiable function of the state vector $|U\rangle$, we estimate the maximum as the value at the location of the mean-state maximum $\left(48^{\circ} \mathrm{N}\right.$ and $612 \mathrm{~m}$ ). This is always true in the linear approach. As such, $\langle F \mid U\rangle$ is a linear operator of $|U\rangle$; hence we can develop it as $\langle F \mid U\rangle=\langle F \mid \bar{U}\rangle+\langle F \mid u\rangle$, where $|\bar{U}\rangle$ is the steady-state vector and $|u\rangle$ is the perturbation-state vector.

\section{a. Optimal pattern and upper bound}

The cost function being defined, the solution expressed in (8) depends only on the delay $\tau$. Optimal perturbations and associated MOC perturbations are computed for several delays from 0.5 to $35 \mathrm{yr}$ (Fig. 2, top right). The growth $\left(\left\langle F|\mathbf{M}(\tau)| u_{0}\right\rangle /\left\langle u_{0}|\mathbf{S}| u_{0}\right\rangle\right)$ is maximal for a delay of $10.5 \mathrm{yr}$. The pattern of the corresponding optimal initial SSS perturbation is localized in the Irminger, Greenland, and Arctic Seas (Fig. 2, left). Introducing this perturbation in the linear model, the time evolution of the MOC intensity confirms a maximum after $10.5 \mathrm{yr}$ (Fig. 2, bottom right).

To validate the linear approach, we have compared the linear and nonlinear time integrations initialized by the linear optimal SSS perturbation for different amplitudes obtained by multiplying the solution shown in Fig. 2 (which peaks at 1 psu) by factors ranging from -20 to 20 (because the initial perturbation is initially 

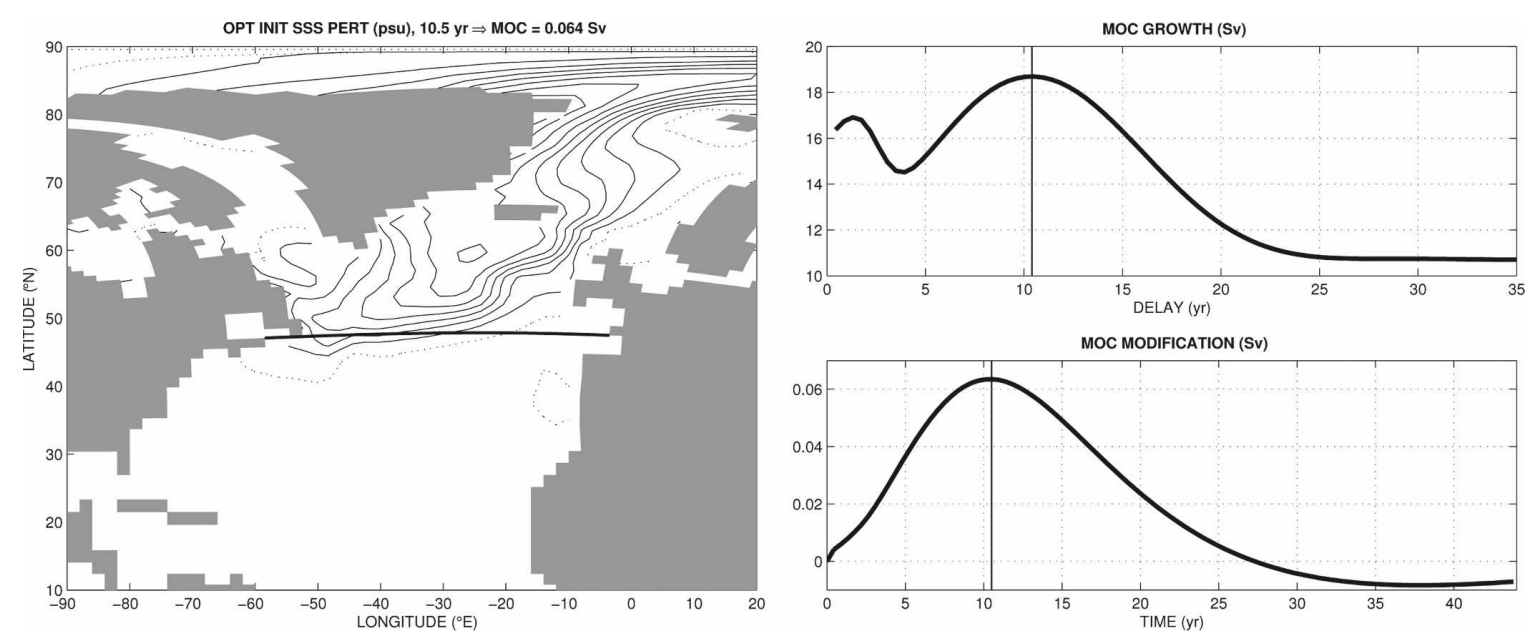

FIG. 2. (left) SSS perturbation, conserving the total amount of salt and modifying most efficiently the intensity of the Atlantic MOC after $10.5 \mathrm{yr}$. The optimal perturbation has been rescaled to a maximum amplitude of 1 psu for clarity. The solid and dotted lines correspond to the positive and zero anomalies; contour interval is $0.1 \mathrm{psu}$. The latitude of the maximum of the mean MOC is denoted by the solid thick line. (top right) Growth of the MOC $\left(\left\langle F|\mathbf{M}(\tau)| u_{0}\right\rangle /\left\langle u_{0}|\mathbf{S}| u_{0}\right\rangle\right)$ as a function of the delay $\tau$ of the transient growth: a maximum appears for a delay of $10.5 \mathrm{yr}$ (vertical line). (bottom right) MOC intensity during the time integration of the linear model initialized by the optimal SSS perturbation (scaled for a 1 psu maximum, corresponding to $\left\langle u_{0}|\mathbf{S}| u_{0}\right\rangle=1.2 \times 10^{-5}$ ): a maximal transient growth appears after $10.5 \mathrm{yr}$ (vertical line), corresponding to a modification of the MOC intensity of $0.064 \mathrm{~Sv}$.

confined in the 10-m-thick upper layer, the actual salinity anomaly decays very rapidly in the much thicker mixed layer). The results (Fig. 3) show that relative differences in MOC response are between $4 \%$ and $58 \%$. Nevertheless, the nonlinear integrations show an asymmetry between positive and negative perturbations, which increases with the perturbations' amplitude. This asymmetry, which cannot be represented in our linear approximation, may correspond to the feed-

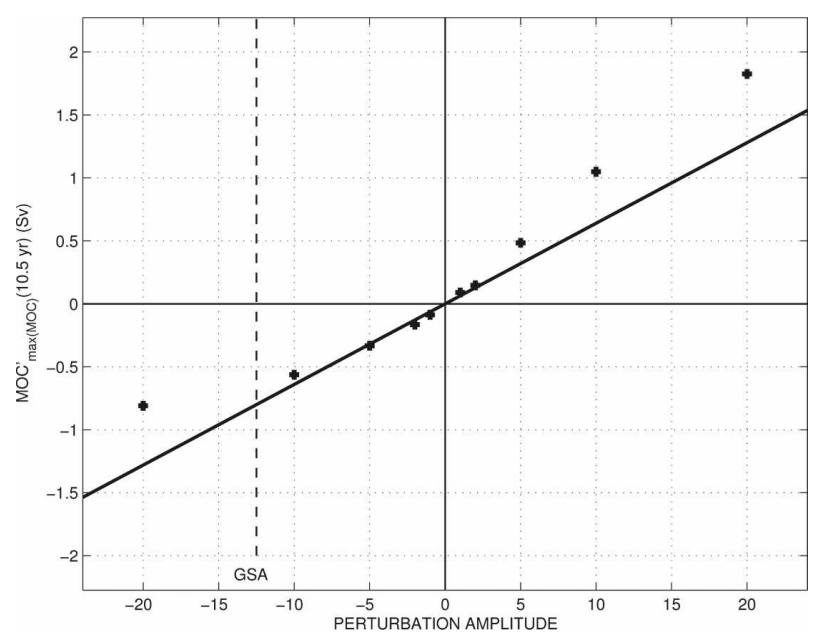

FIG. 3. Optimal growth of the MOC intensity, as a function of the optimal initial perturbation amplitude (maximum SSS anomaly in psu), according to the linear (solid line) and the nonlinear time integrations (crosses). The vertical dashed line corresponds to a typical amplitude of GSA (Belkin et al. 1998). back of density anomalies on the vertical mixing. Indeed, a positive anomaly will enhance the vertical mixing, hence the deep water formation, resulting in a stronger MOC than in the linear model; whereas, a negative anomaly will decrease the vertical mixing, hence the deep water formation, resulting in a weaker MOC compared to the linear model. Note that this comparison ensures that the nonlinear evolution of the linear optimal perturbation can be approximated by the linear model in this amplitude range, but it does not allow us to verify that this pattern is the optimal perturbation in the full nonlinear problem (Mu et al. 2004).

Because we have identified the optimal SSS perturbation, no other SSS perturbation can impact the MOC intensity more than in the linear model. In other words, this analysis permits us to set an upper bound for the MOC intensity modification in the linear model under any SSS perturbation. Analyses of the Great Salinity Anomalies (GSA; Belkin et al. 1998) reveal SSS maximum modification in the North Atlantic of about 0.5 psu over a $250-\mathrm{m}$-thick layer. For such perturbations (corresponding to a 12.5-psu maximum salinity anomaly initially confined in the 10-m-thick upper layer), we would obtain a modification of the MOC intensity of $0.8 \mathrm{~Sv}$, that is, $11.5 \%$ of our mean circulation. For such amplitude, the linear approximation induces an error less than $14 \%$ (Fig. 3).

At this stage of our analysis, a question remains: How does the SSS perturbation initially located in the north of the basin modify the MOC at $48^{\circ} \mathrm{N} 10 \mathrm{yr}$ later? 


\section{b. Growth mechanism}

To understand the physical mechanisms inducing the finite-time growth, we focus on the anomaly fields at the time of the maximum growth (Fig. 4). As a first step, a rescaling of the perturbations allows us to better understand the dynamical regime of the evolving anomalies. We obtain the following values for the Rossby number (Ro) and the horizontal and vertical Ekman numbers $\left(\mathrm{Ek}_{\mathrm{H}}\right.$ and $\left.\mathrm{Ek}_{\mathrm{V}}\right)$ :

$$
\begin{aligned}
\mathrm{Ro} & =\frac{\bar{U}}{f L} \simeq 10^{-3} \ll 1, \\
\mathrm{Ek}_{\mathrm{H}} & =\frac{v_{H}}{f L^{2}} \simeq 10^{-2} \ll 1, \\
\mathrm{Ek}_{\mathrm{V}} & =\frac{v_{\mathrm{V}}}{f H^{2}} \simeq 1,
\end{aligned}
$$

where $\bar{U} \simeq 10^{-2} \mathrm{~m} \mathrm{~s}^{-1}$ is the scaling of the mean velocity (Ro is the ratio of the advection-nonlinearterm and the Coriolis term; in its expression for the linear equations, the scaling of the mean velocity appears); Here, $L \simeq 10^{5} \mathrm{~m}\left(H \simeq 10^{3} \mathrm{~m}\right)$ is the scaling of the horizontal (vertical) anomalies' length; $f=10^{-4} \mathrm{~s}^{-1}$ is the Coriolis parameter; and $v_{\mathrm{H}(\mathrm{V})} \simeq 10^{4}\left(10^{2}\right) \mathrm{m}^{2} \mathrm{~s}^{-1}$ is the horizontal (vertical) turbulent viscosity coefficient. This scaling shows that the nonlinear linearized terms and the horizontal turbulent viscosity are negligible in comparison with the Coriolis term, but the vertical turbulent viscosity may have the same magnitude as the Coriolis term. Nevertheless, while $\nu_{\mathrm{V}}$ is of the order of $100 \mathrm{~m}^{2} \mathrm{~s}^{-1}$ in few localized areas (lateral boundary currents like the Gulf Stream), it is generally two orders of magnitude weaker almost everywhere. Because the anomalies are not localized only at the boundary of the basin, we assume in the following that the vertical turbulent viscosity is also negligible compared to the Coriolis term. The regime can thus be considered as geostrophic, such that the velocities are parallel to the density contours, as mostly confirmed in Fig. 4.

In such a diagnostic geostrophic dynamical regime, we can limit our investigation to the evolution of the temperature and salinity anomaly fields. It appears that the zonal density gradient anomaly is mainly controlled by the temperature (zonal gradients of salinity anomaly are weaker, $\alpha \partial_{\phi} \mathrm{SST}^{\prime} / \beta \partial_{\phi} \mathrm{SSS}^{\prime} \simeq 5$ ), whereas the meridional density gradient anomaly is controlled by the salinity (meridional gradients of temperature anomaly are weaker, $\alpha \partial_{\theta} \mathrm{SST}^{\prime} / \beta \partial_{\theta} \mathrm{SSS}^{\prime} \simeq 0.5$ ) around $48^{\circ} \mathrm{N}$. Hence, the increase of the MOC intensity is mainly due to the zonal gradient of temperature anomaly. However, the initial perturbation is a positive salinity anomaly in the north of the Atlantic basin, inducing a meridional density gradient of salinity. At this stage of the analysis, we still have to understand how the initial anomaly of salinity has induced a zonal gradient of temperature, like the one present at $10.5 \mathrm{yr}$, resulting in an increased MOC intensity.

Before studying the growth mechanism, a more detailed description of the mean circulation is required. Along $48^{\circ} \mathrm{N}$, the temperature dominates the zonal density gradient by a factor of 2 (Fig. 5, left). This temperature profile can be sketched as an eastward increase followed by a weak decrease near $20^{\circ} \mathrm{W}$ (Fig. 5 , top left), the latter feature being absent in the mean salinity.

The initial salinity perturbation induces a positive anomaly of the surface meridional density gradient at $48^{\circ} \mathrm{N}$, producing a positive vertical shear in zonal velocity according to the thermal wind relation. Assuming a two-layer vertical structure, thermal wind results in a positive zonal velocity anomaly at the surface (Fig. 5, right, dotted line). By interacting with the zonal temperature gradient of the steady state, the zonal velocity anomaly creates a trend of temperature negative east of $\sim 20^{\circ} \mathrm{W}$ and positive west of $\sim 20^{\circ} \mathrm{W}$, given the concave shape of the zonal profile of the mean temperature (a positive gradient west of $20^{\circ} \mathrm{W}$ and a negative gradient east of $20^{\circ} \mathrm{W}$ ), hence the buildup of a positive zonal temperature gradient (Fig. 5, left, dashed line).

After $10.5 \mathrm{yr}$, the zonal density gradient is largely dominated by the temperature gradient. According to the thermal wind relation, the appearance of a positive zonal temperature gradient induces a positive meridional shear anomaly, hence a northward velocity anomaly in the surface layer (Fig. 5, right, dashed line) and a southward velocity in the deep ocean.

In the two-layer simplification, this baroclinic anomaly induces an MOC anomaly with a streamfunction maximum at the interface simply proportional to the upper velocity anomaly. In conclusion, the appearance of a positive zonal surface temperature gradient leads to an increase of the MOC with a linear relationship between the two.

Given the mean state, we have obtained linear relationships between the anomalies of the meridional salinity gradient and the MOC intensity through anomalies of the zonal temperature gradient, hence a direct linear relationship between the SSS anomaly in the north of the Atlantic basin and the MOC intensity. Attempts to estimate these relationships quantitatively through analytical formulas lead to a reasonable evaluation of the order of magnitude of the response.

After this 10.5-yr transient growth, a decrease of the MOC intensity appears (Fig. 6). This decrease is asso- 

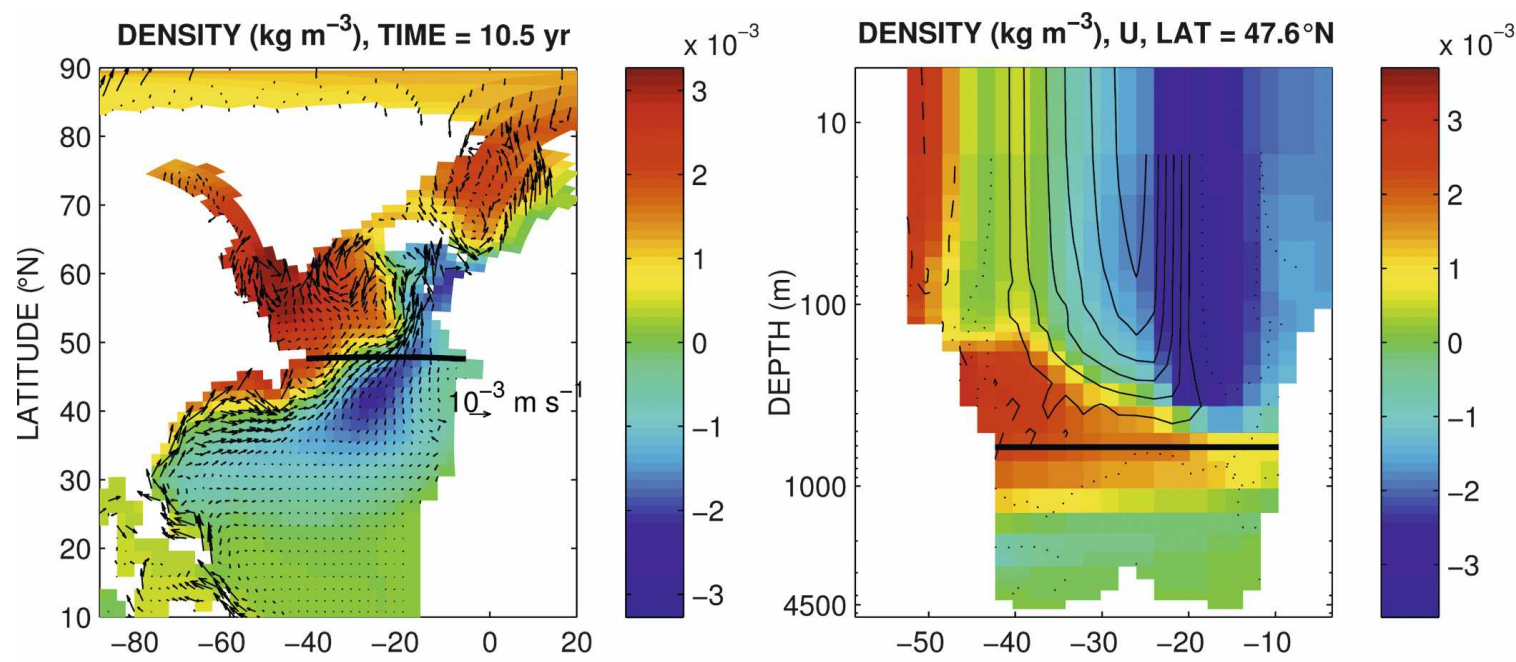

TEMPERATURE ( $\mathrm{C}$ ), Z-MEAN = $0-612 \mathrm{~m}$
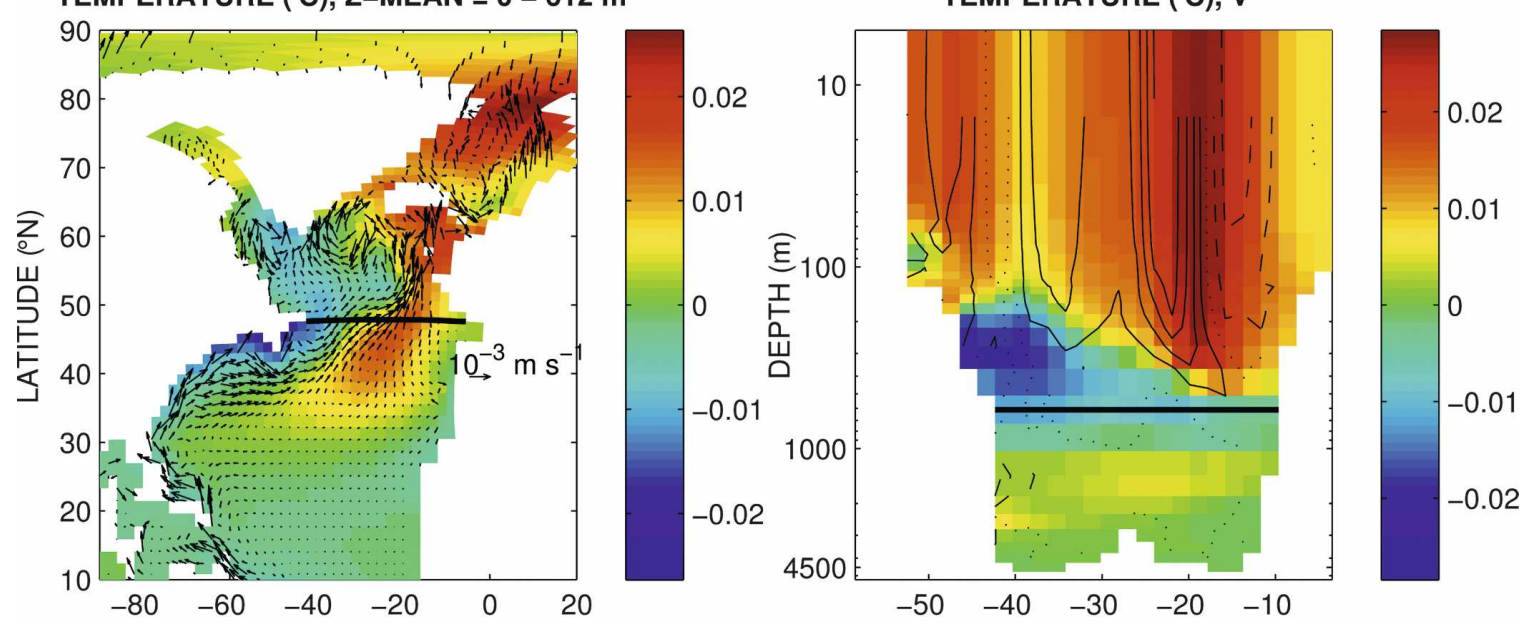

TEMPERATURE (C), V
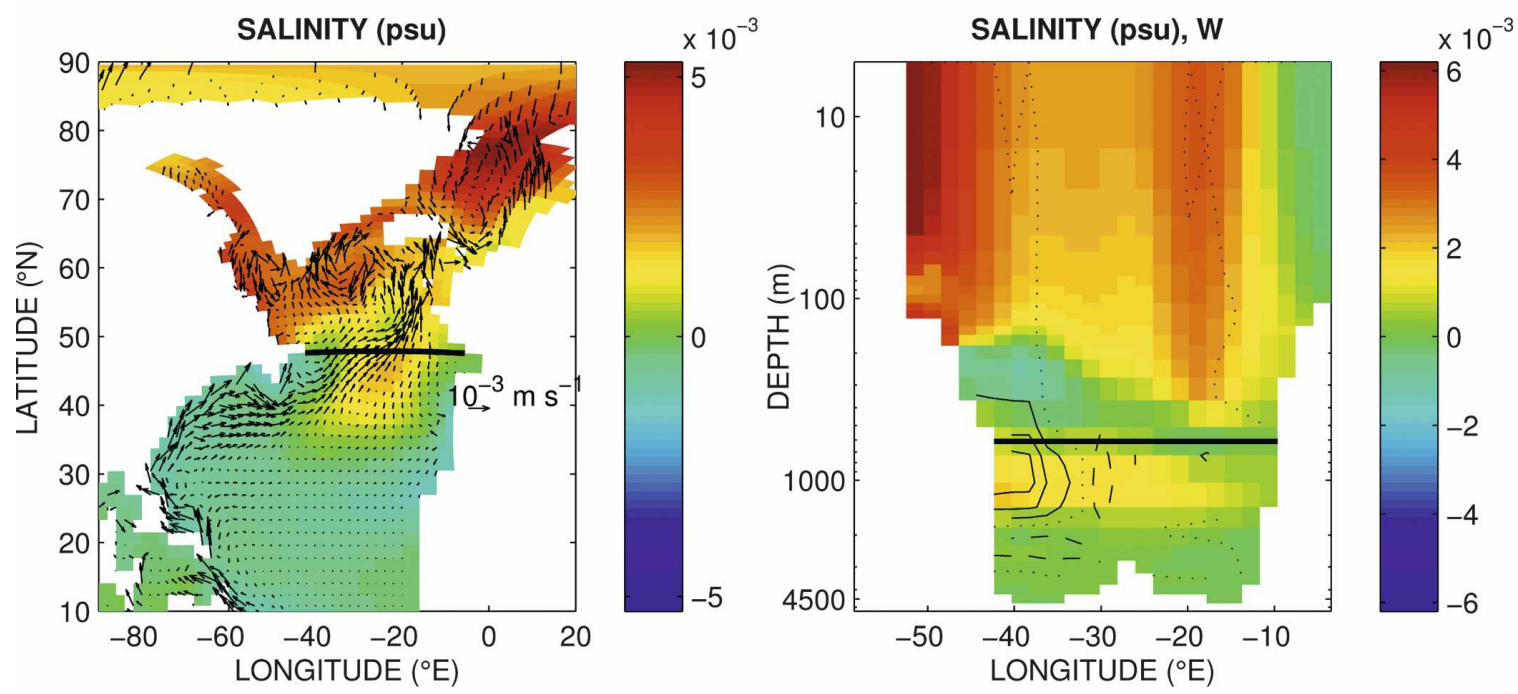

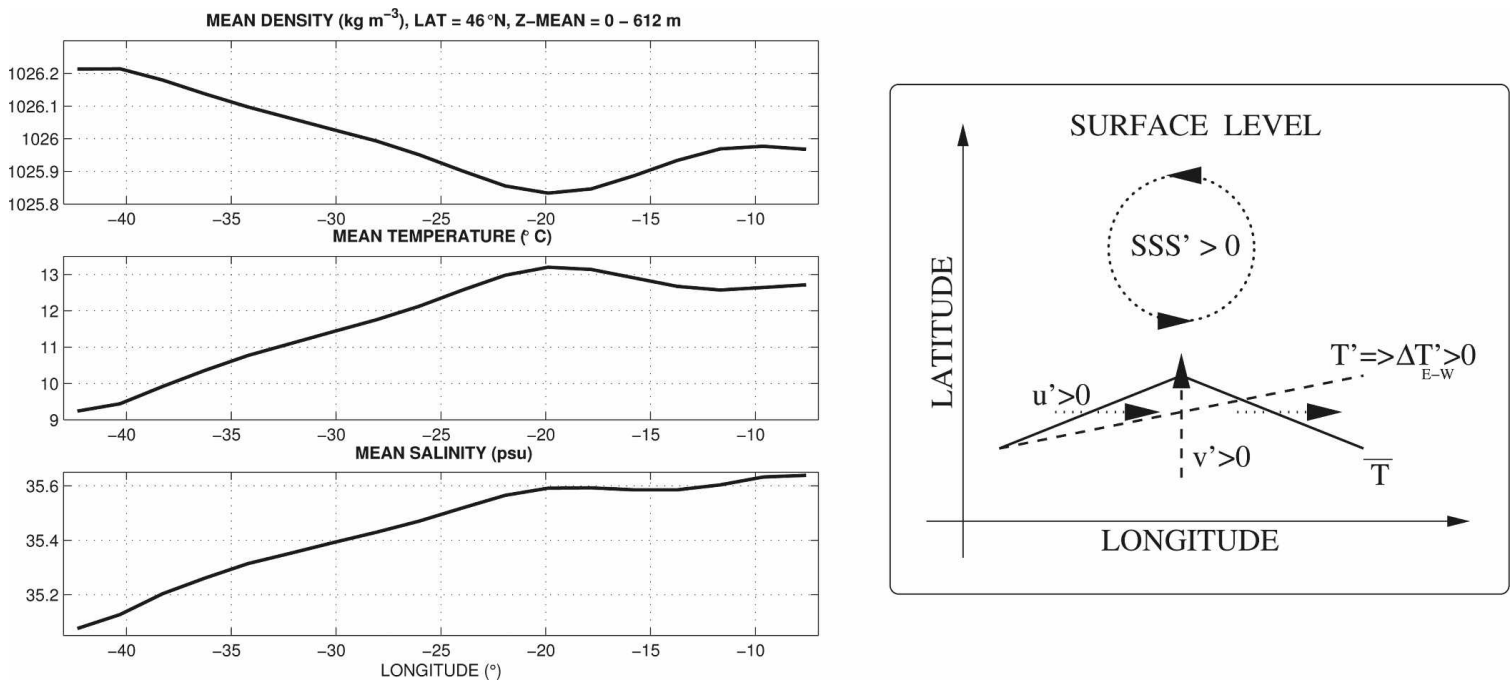

FIG. 5. (left) Mean density, temperature, and salinity along $48^{\circ} \mathrm{N}$ in the Atlantic. The density gradient is dominated by the temperature along this section. The temperature profile can be sketched as an inverse U shape along the section. (right) Mechanistic sketch allowing a North Atlantic positive SSS anomaly to increase the northward surface advection, which thus enhances the MOC intensity. The dotted, dashed, and solid lines represent the salinity anomaly and associated surface zonal velocity, the isothermal contour of the anomaly and the associated surface meridional velocity, and the isothermal contour of the mean state.

ciated with an increase of the temperature in the North Atlantic. Actually, the increase of the northward surface velocities brings warm water in the north region and progressively modifies the North Atlantic SST anomaly. This increase of SST in the north reverses the meridional surface density gradient of the initial perturbation. Thus, by the opposite mechanism than during the transient growth (Fig. 5), the MOC intensity decreases. This process is known as the negative temperature feedback on MOC intensity (Marotzke 1996).

After the MOC intensity reaches zero, a significant negative phase appears. This negative phase reaches a maximum at $38 \mathrm{yr}$. At this time, the temperature pattern is close to the opposite of the one at $10.5 \mathrm{yr}$ (time of the transient growth maximum). Then, after $38 \mathrm{yr}$, the temperature anomaly in the north region decreases due to the inversion of the surface velocities and thus of heat transport (this is also the mechanism of negative temperature feedback). However, the existence of these two opposite phases suggests that this transient growth may be due to the stimulation by the SSS per- turbation of a damped oscillatory mode dominated by temperature. A crude estimation based on these two opposite phases gives us a period of $\sim 55 \mathrm{yr}$ for a damping time scale of $\sim 13 \mathrm{yr}$. The thermal structure of this oscillation looks similar to the 50 -yr oscillation found in the Geophysical Fluid Dynamics Laboratory (GFDL) ocean-atmosphere coupled model (Fig. 19 of Delworth et al. 1993), whereas the density structure resembles the 25-yr oscillation found in the third climate configuration of the Met Office Unified Model (HadCM3) ocean-atmosphere sea ice coupled model (Dong and Sutton 2005; Fig. 8). These works and ours may support the existence of an oceanic damped oscillatory mode that is able to modify the MOC through a nonnormal process.

\section{Optimal perturbation for the meridional heat transport}

We now use the method applied in the previous section to study the optimal perturbation of the merid-

$\leftarrow$

FIG. 4. Snapshot at $10.5 \mathrm{yr}$ of the linear model time integration initialized by the optimal SSS perturbation for the MOC intensity. (left) Density, temperature, and salinity perturbations, with the anomalous horizontal velocity field superimposed, all averaged from 0 to $612 \mathrm{~m}$ (depth of the mean MOC maximum). (right) The $48^{\circ} \mathrm{N}$ section (latitude of the mean MOC maximum) of density, temperature, and salinity, respectively, with the contoured field of zonal, meridional, and vertical velocities (the latter is averaged over $20^{\circ}$ of latitude). The solid, dashed, and dotted lines correspond to the positive, negative, and zero anomalies; horizontal and vertical velocity contour intervals are $5 \times 10^{-5} \mathrm{~m} \mathrm{~s}^{-1}$ and $5 \times 10^{-9} \mathrm{~m} \mathrm{~s}^{-1}$, respectively. The thick solid line denotes the latitude and depth of the mean MOC maximum. 

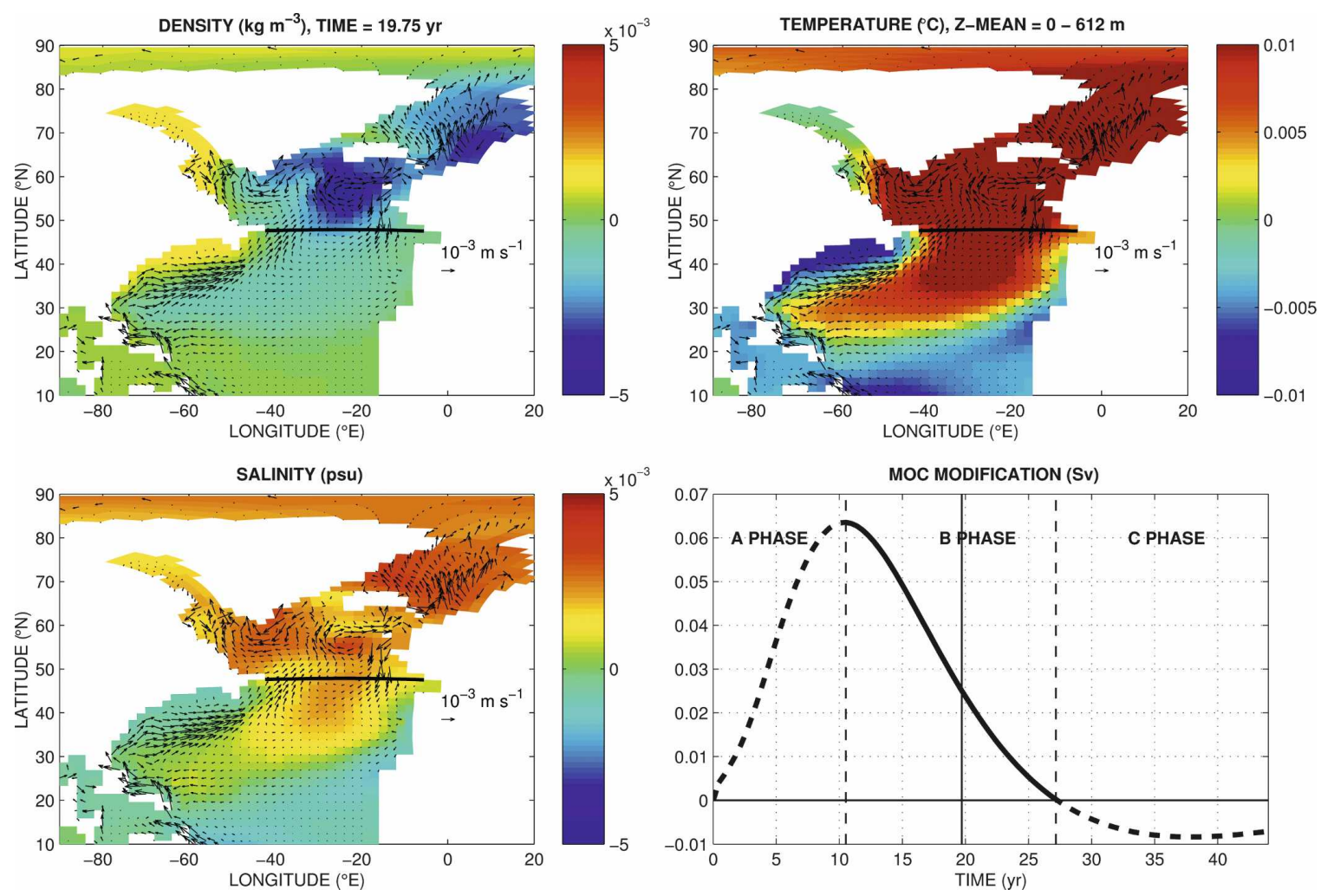

FIG. 6. Same as in Fig. 4 (left), but at 19.75 yr. (bottom right) MOC intensity as a function of time (the vertical solid line shows the snapshot time), showing the transient growth phase A, the decay phase B (bold solid line limited by the two vertical dashed lines), and the opposite phase $\mathrm{C}$.

ional heat transport. The difference appears only in the definition of function $\langle F|$ in $(8)$, which is now the maximum of the meridional heat transport. The MHT is not a linear operator of the state vector, because it is a product of meridional velocity and temperature:

$$
\mathrm{MHT}=\rho_{0} C_{p} \int_{\phi_{\mathrm{west}}}^{\phi_{\mathrm{east}}} a \cos \theta d \phi \int_{-H}^{0} d z v T,
$$

where $C_{p}$ is the heat capacity of seawater. Using overbars for the mean state and primes for the perturbations, we obtain

$$
\mathrm{MHT}=\rho_{0} C_{p} \int_{\phi_{\text {west }}}^{\phi_{\mathrm{east}}} a \cos \theta d \phi \int_{-H}^{0} d z\left(\bar{v} \bar{T}+\bar{v} T^{\prime}+v^{\prime} \bar{T}+v^{\prime} T^{\prime}\right)=\overline{\mathrm{MHT}}+\mathrm{MHT}^{\prime}+\mathrm{MHT}^{\prime \prime}
$$

The MHT can be separated into a mean term, a firstorder term, and a second-order term in the perturbation amplitude. Neglecting the latter, we obtain the linearized MHT anomaly

$$
\mathrm{MHT}^{\prime}=\rho_{0} C_{p} \int_{\phi_{\mathrm{west}}}^{\phi_{\mathrm{east}}} a \cos \theta d \phi \int_{-H}^{0} d z\left(\bar{v} T^{\prime}+v^{\prime} \bar{T}\right)
$$

We now take $\langle F \mid u\rangle$ as the $\mathrm{MHT}^{\prime}$ value at the latitude of the mean MHT maximum, that is, $27^{\circ} \mathrm{N}$ (Fig. 1). Our new cost function is defined as $\operatorname{MHT}^{\prime}\left(\theta=27^{\circ} \mathrm{N}\right)$ and is used in (8) to compute the optimal perturbation and the
MHT growth for several delays $\tau$ ranging from 0.2 to $22 \mathrm{yr}$.

\section{a. Optimal pattern and upper bound}

The maximum impact on $\mathrm{MHT}^{\prime}$ appears for a delay of $2.2 \mathrm{yr}$ (Fig. 7, top right). We did not consider the very first peak around a 6-month delay because the associated optimal pattern is very noisy: we chose to address only the second peak when diffusion had time to smooth the patterns, and select the large spatial scale, which is of relevance to climate. The pattern of SSS that conserves salt and modifies most efficiently the MHT 

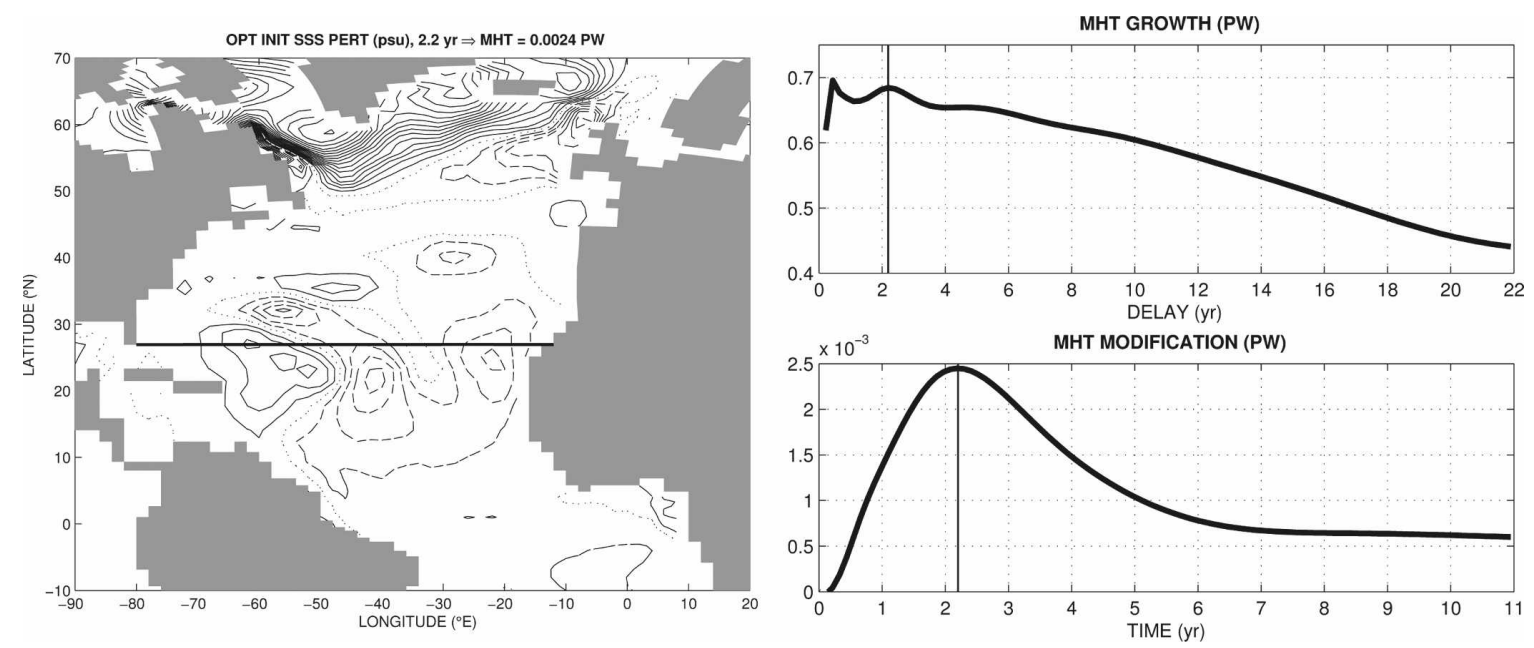

FIG. 7. Same as in Fig. 2, but for the optimal SSS perturbation of MHT. The thick line denotes the latitude of the mean MHT maximum. The contour interval is $0.05 \mathrm{psu}$. The maximum growth appears for a delay of $2.2 \mathrm{yr}$ and induces a modification of $0.0024 \mathrm{PW}$.

intensity corresponds to a strong anomaly in the Labrador and Irminger Seas and another anomaly, one order of magnitude weaker, composed of two main lobes, located between $15^{\circ}$ and $30^{\circ} \mathrm{N}$ in the west of the Atlantic basin (Fig. 7, left). This latter anomaly changes sign between $60^{\circ}$ and $35^{\circ} \mathrm{W}$. The linear model integration initialized by this optimal SSS anomaly reveals a transient growth reaching its maximum after $2.2 \mathrm{yr}$ (Fig. 7, bottom right). This maximum corresponds to an MHT modification of $2.4 \times 10^{-3} \mathrm{PW}$ as predicted by the solution (8). The associated MHT anomaly is mainly driven by the $v^{\prime} \bar{T}$ term, which is two orders of magnitude greater than $\bar{v} T^{\prime}$. It thus appears that the SSS optimal perturbation modifies more efficiently the MHT through velocity anomalies advecting the mean temperature than temperature anomalies advected by the mean meridional velocity.

Again, for validation purposes, we compared linear and nonlinear time integrations of the optimal SSS perturbation with different amplitudes ranging from -20 to 20 (Fig. 8) and found relative differences in MHT response lying between $4 \%$ and $45 \%$. The asymmetry between positive and negative anomalies in the nonlinear time integration is not as important as before, because now the perturbations are not located only in the deep water formation regions; hence convection, which is poorly represented in the tangent linear model, plays a weaker role.

As in the previous section, we also establish an upper bound of the MHT modification. A perturbation of the order of the Great Salinity Anomalies cannot modify the MHT intensity by more than $0.03 \mathrm{PW}$ (i.e., $5.3 \%$ of the mean state). For this amplitude, the linear approximation induces an error less than $22 \%$ (Fig. 8).

\section{b. Growth mechanism}

We proceed now to the description of the mechanism driving the MHT increase by the optimal SSS perturbation. As for the study of the MOC intensity modification, the dynamical regime, at the maximum of the transient growth $(2.2 \mathrm{yr})$, corresponds to $\mathrm{Ro} \ll 1$, $\mathrm{Ek}_{\mathrm{H}} \ll 1$ and $\mathrm{Ek}_{\mathrm{V}} \sim 1$. For the same reason, we will neglect the vertical turbulent viscosity compared to the Coriolis term (Fig. 9). We can thus suppose that the flow is geostrophic.

After $2.2 \mathrm{yr}$, the positive salinity anomaly in the Labrador Sea is balanced by a positive thermal anomaly in terms of density; hence, no velocity anomalies occur. As a consequence, the increase of the MHT intensity cannot be explained by the salinity anomaly in the Lab-

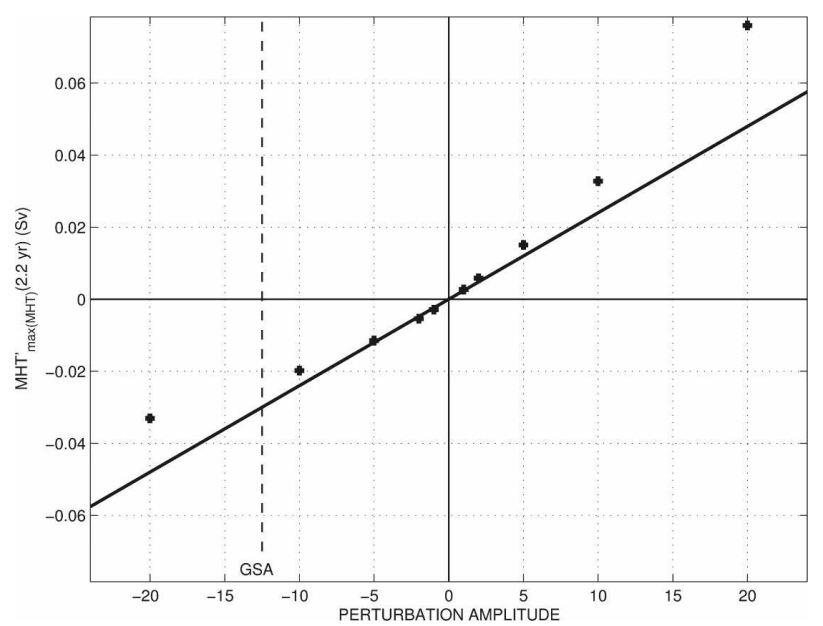

FIG. 8. Same as in Fig. 3, but for the MHT. 

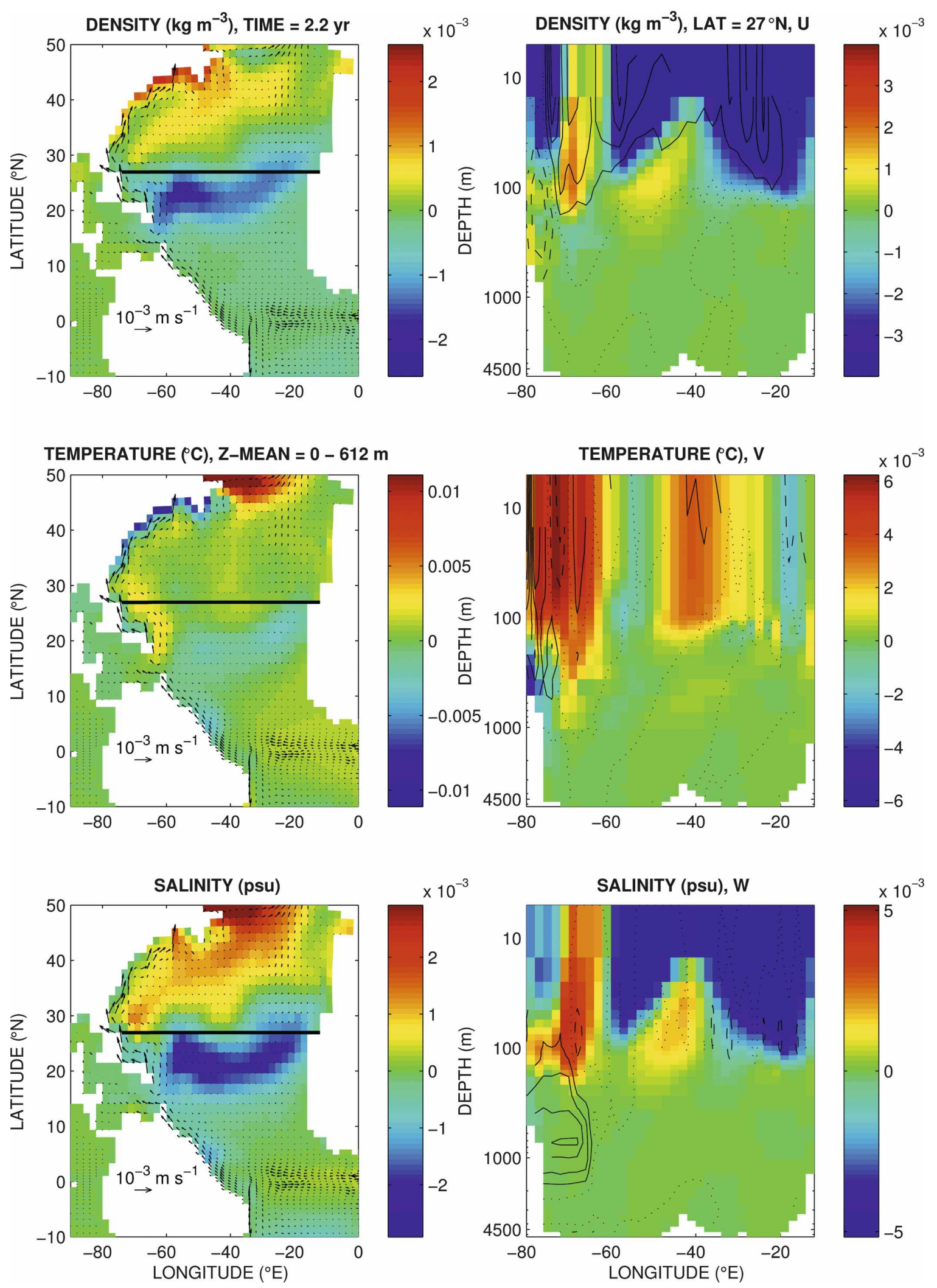

FIG. 9. Same as in Fig. 4, but for snapshots at $2.2 \mathrm{yr}$ of the linear model time integration initialized by the optimal SSS perturbation of MHT. The thick solid line denotes the latitude of the mean MHT maximum. (left) The average is from 0 to $612 \mathrm{~m}$ (maximum depth of the anomalies). (right) The $27^{\circ} \mathrm{N}$ section (latitude of the mean MHT maximum). The horizontal and vertical velocity contour intervals are $5 \times 10^{-5} \mathrm{~m} \mathrm{~s}^{-1}$ and $5 \times 10^{-9} \mathrm{~m} \mathrm{~s}^{-1}$, respectively. 

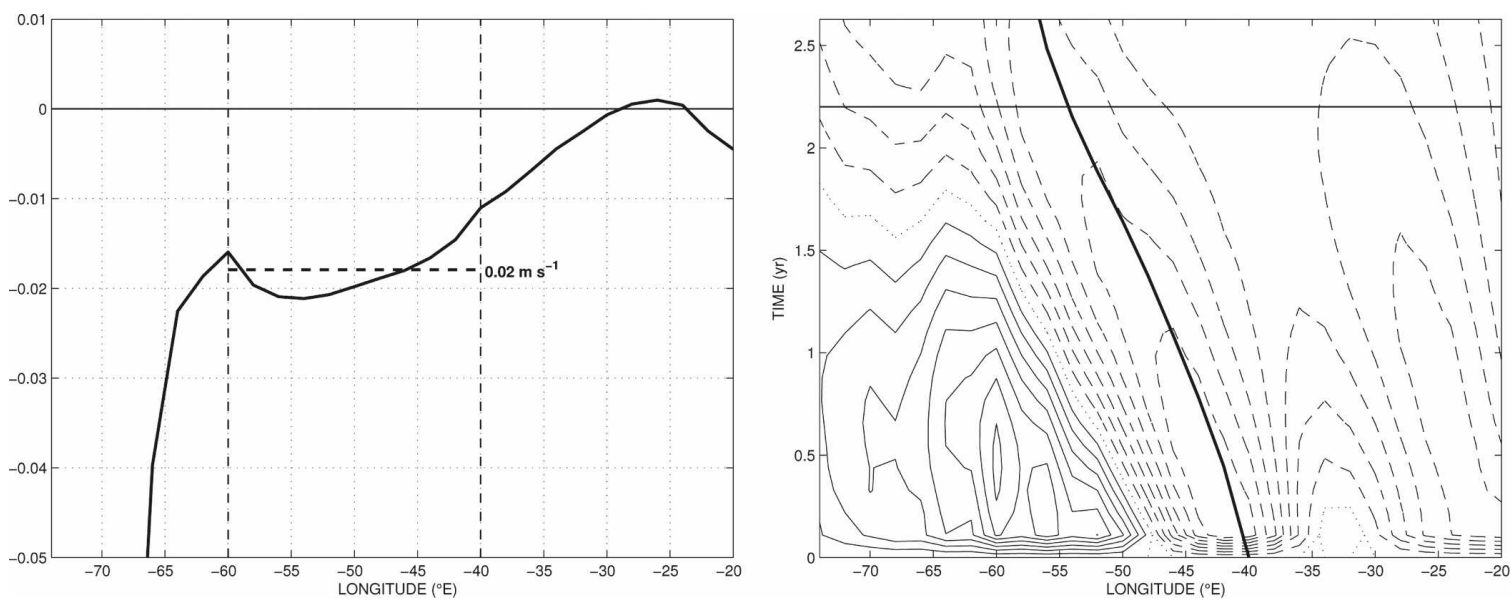

FIG. 10. (left) Zonal velocity profile of the mean state averaged from 0 to $197 \mathrm{~m}$ (local maximum depth of the anomaly) at $23^{\circ} \mathrm{N}$ (south of the mean MHT maximum). The zonal mean advection between $40^{\circ}$ and $60^{\circ} \mathrm{W}$ is around $2.2 \mathrm{~cm} \mathrm{~s}^{-1}$. (right) Hovmoller of the density anomalies averaged from 0 to $197 \mathrm{~m}$ along $23^{\circ} \mathrm{N}$, for the linear model time integration initialized by the optimal SSS perturbation (normalized to a 1 psu maximum). The solid, dashed, and dotted lines correspond to the positive, negative, and zero anomalies; contour interval is $10^{-3} \mathrm{~kg} \mathrm{~m}^{-3}$. The propagation of the anomalies is due to the mean zonal advection (solid thick line). The horizontal solid line denotes the maximum growth time for the MHT (2.2 yr).

rador Sea. It appears that the increased MHT is due to increased meridional velocities in the western boundary. These meridional velocities are orthogonal to the zonal gradient of temperature anomaly, also located in the west of the Atlantic basin (see Fig. 9 for vertical section between 100 and $1000 \mathrm{~m}$ ).

At this stage of the analysis, it seems that as seen above, the meridional velocity anomalies are induced by the zonal gradient of the temperature anomaly after $2.2 \mathrm{yr}$, which has been induced by the initial salinity anomaly. We thus proceed to identify how the initial SSS anomaly has been able to increase the MHT $2 \mathrm{yr}$ later through the zonal gradient of temperature anomaly at $27^{\circ} \mathrm{N}$.

The initial SSS anomaly corresponds to a zonal gradient between $60^{\circ}$ and $35^{\circ} \mathrm{W}$ around $27^{\circ} \mathrm{N}$. This anomaly propagates during $2.2 \mathrm{yr}$ before the MHT reaches its maximum. This propagation is mainly due to the mean transport (Fig. 10, right). Moreover, because the velocities are one order of magnitude stronger in the south $\left(\sim 2.2 \mathrm{~cm} \mathrm{~s}^{-1}\right.$ westward, see Fig. 10, left) than in the north $\left(\sim 0.5 \mathrm{~cm} \mathrm{~s}^{-1}\right.$ westward $)$ of $27^{\circ} \mathrm{N}$, the mean transport turns the salinity anomaly gradient to create, after $2.2 \mathrm{yr}$, a positive meridional gradient of salinity anomaly around $27^{\circ} \mathrm{N}$.

Because the salinity anomaly results in a density anomaly, the rest of the analysis is similar to the one conducted in section $3 \mathrm{~b}$. By the thermal wind relation, an eastward surface velocity appears. The mean-state temperature first increases eastward, then decreases, but it is now localized at the western boundary of the basin. Accordingly, the interaction of the zonal anomaly of advection with the gradient of the mean temperature induces a localized temperature anomaly on the western boundary. The anomaly consists of a positive zonal gradient of temperature.

According to the thermal wind relation, the positive zonal gradient of the temperature anomaly creates a positive meridional shear, which corresponds to a northward surface flow compensated for by a deep southward flow. Again considering the ocean as a twolayer system, the MHT anomaly is proportional to the upper velocity anomaly multiplied by the difference in mean temperature between the upper and lower layers.

We have derived the linear relationship between the meridional gradient of the salinity anomaly and the zonal gradient of the temperature anomaly, another one between the zonal gradient of temperature anomaly and the anomaly of meridional surface velocities, and finally the relation between the anomaly of meridional surface velocities and the MHT intensity anomaly. We have thus established a direct positive linear relationship between the meridional gradient of the salinity anomaly and the MHT intensity anomaly. A more accurate formulation of these relationships provides a quantitative estimation of the MHT response to the salinity perturbation gradient with an order of magnitude comparable to the linear model response.

After this 2.2-yr growth of MHT, the negative SSS anomaly is advected into the western boundary current and moves rapidly northward, away from $27^{\circ} \mathrm{N}$. This reduces the anomalous MHT intensity, and the optimal transient growth ceases. After this advective process, a slow decrease due to diffusion takes place. 


\section{Discussion and conclusions}

In the context of global warming, significant modifications of surface freshwater flux and sea surface salinity have occurred in the North Atlantic during the past few decades. In this study, we have tried to estimate the variations of the ocean circulation and transport that may result from such modifications.

A maximization method using Lagrangian multipliers has been implemented to obtain optimal initial SSS perturbations. We have chosen to address the maximal response of both the intensity of the meridional overturning circulation (MOC) and the meridional heat transport (MHT), as indicators of the ocean circulation. Moreover, we have imposed three constraints: the perturbation applies to SSS only, the perturbation conserves the total salt content, and the initial perturbation is normalized given our linear framework. This maximization method leads to an explicit solution, as opposed to an eigenvalue problem, for the optimal initial SSS perturbations.

The method is applied to the primitive equation model OPA in the realistic global configuration ORCA2. We have used this model under constant surface boundary conditions (corresponding to the average over a season of all the forcing terms), including restoring for SST and SSS, to obtain a real steady state. Finally, the computation of the optimal perturbations uses the OPA tangent linear and adjoint models. They use prescribed fluxes of heat and freshwater equivalent to the nonlinear model in a steady state, and no relaxation except in the equatorial Pacific.

The first result of this study is the existence of a clear optimal transient growth of the MOC $10.5 \mathrm{yr}$ after the SSS perturbation. The optimal SSS pattern inducing this transient growth corresponds to a modification of the SSS in the Irminger, Greenland, and Arctic seas. A positive (the anomaly sign is not important in a linear context) SSS anomaly in the northern North Atlantic induces a positive meridional density gradient. This gradient creates an eastward surface velocity through the thermal wind relation. This surface velocity advects the mean temperature zonal gradient to produce a positive gradient of the temperature anomaly. The latter creates a northward velocity that intensifies the MOC.

The existence of an optimal transient growth of the MHT is not as obvious: a weak peak exists $2.2 \mathrm{yr}$ after the SSS perturbation, which has been analyzed. The associated optimal SSS pattern corresponds to a zonal gradient localized around $27^{\circ} \mathrm{N}$ (latitude of the mean MHT maximum). We have shown that this zonal gradient rotates under the mean flow advection to produce a meridional gradient after $2.2 \mathrm{yr}$. The resulting merid- ional gradient acts according to the mechanism described above to increase the northward surface flow. This velocity, localized in the western boundary, reinforces the MHT.

These two experiments allowed us to test two different measures. The main difference occurring during our work is that the two measures do not lead to the same time scale. Our MOC experiment shows that the time scale is $10.5 \mathrm{yr}$ and the transient growth mechanism is mainly due to the interaction between the perturbation and the large-scale zonal gradient of temperature. For the MHT experiment, the time scale is $2.2 \mathrm{yr}$ and the transient growth mechanism is due to passive advection of the perturbation by the mean flow. The differences are mainly due to ocean circulation characteristics at the latitude of the measure $\left(48^{\circ} \mathrm{N}\right.$ for the MOC; $24^{\circ} \mathrm{N}$ for the MHT).

The main result is the determination of upper bounds of variability of the MOC or MHT in response to surface salinity anomalies. Indeed, because we have found the optimal perturbations, then in the linear limit, no other perturbations can alter the ocean circulation more. Given that the Great Salinity Anomalies correspond to a modification of about 0.5 psu over the upper $250 \mathrm{~m}$, we obtain that the MOC cannot be modified by more than $0.8 \mathrm{~Sv}$ ( $11 \%$ of the mean circulation) and the MHT by more than 0.03 PW (5\% of the mean circulation) according to our model. For the sake of comparison, Häkkinen (1999) shows that an idealistic GSA initial perturbation leads to a strong modification corresponding to $20 \%$ for the MOC and $17 \%$ for the MHT in a coupled ocean-ice model. In contrast, Haak et al. (2003) reproduce the chain of processes that led to the formation and propagation of the 1970s, 1980s, and 1990s GSAs in the same kind of model. In such a setup, the GSA alters the MOC by less than $2 \%$ and the MHT by $5 \%$. The impact of a GSA on the ocean circulation seems, therefore, crucially dependent on the time scale on which ice pulses are injected in the ocean. Our results are in better agreement with those of the most recent study.

This study can be extended in several directions. First, one can consider optimal perturbations of SST or wind stress. Preliminary results confirm our interest in SSS perturbations. Indeed, the optimal pattern of SST (not shown) looks very similar to the one of SSS, but to obtain an equivalent modification of the MOC, the temperature needs to be modified by more than $5^{\circ} \mathrm{C}$ on $250 \mathrm{~m}$, which does not sound very realistic. However, because we search the optimal perturbation, if we allow a perturbation of both SSS and SST (and wind stress if needed), a constructive pattern will lead to a more ef- 
ficient modification of the MOC than can be achieved with only an SSS perturbation.

Another direction would be to take into account the full seasonal cycle influencing the forcing and the ocean circulation, instead of the steady situation considered herein, and solve the associated nonautonomous problem. Then our mean overturning circulation and associated poleward heat transport would certainly be more intense, hence closer to observations. This would certainly lead to shorter optimal delays because of faster advection processes. One could also expect that a stronger overturning would be less sensitive to salinity perturbations (Tziperman 1997). This would also clarify the question of the sensitivity of the MOC to SSS perturbations as a function of the season (the seasonal cycle strongly modulates the mixed layer depth, i.e., hence the vertical mixing of surface perturbations).

Another direction is to add an atmospheric model and a sea ice model as a next step toward a more realistic representation of the water cycle. This would allow a better understanding of climate feedbacks and of the impact of freshwater on the oceanic circulation, and thus will modify our optimal perturbation patterns.

We have shown that the linear model can represent accurately the evolution of the optimal perturbations, for a realistic range of amplitudes, through comparisons with the nonlinear evolution. However, we have not taken into account the full nonlinear dynamics in the search for the optimal perturbations, in contrast with studies on the conditional nonlinear optimal perturbation. This kind of method would permit us to see the asymmetry between positive and negative perturbations and to determine the sensitivity to the intensity of the perturbations. Nevertheless, this has only been done in idealized ocean circulation models so far, as in Stommel's (1961) two-box model (Mu et al. 2004), a coupled ocean-atmosphere box model (Sun et al. 2005), and in a two-dimensional quasigeostrophic model (Mu and Zhang 2006).

But the most important step toward the understanding of the optimal growth mechanism (the maximum growth delay is clearly not rationalized here, i.e.) would be to obtain the eigenmodes of the linear and adjoint models. This analysis will (or will not) validate the existence of a damped 55-yr oscillatory mode that seems to be triggered by the optimal SSS perturbation in our analysis. Moreover, this last step will allow us to obtain the optimal constant perturbation, the optimal stochastic perturbation, and the associated spectral response as derived in Sévellec et al. (2007). This would enable us to better understand the response mechanisms of our oceanic model and its preferential modes of variability.
Acknowledgments. Computations have been partly performed on the NEC vectorial machine at the CNRS national computing center (IDRIS, Orsay, France). Several comments and suggestions from Michael Ghil and Andy Moore are gratefully acknowledged.

\section{REFERENCES}

Arzel, O., T. Huck, and A. C. de Verdière, 2006: The different nature of the interdecadal variability of the thermohaline circulation under mixed and flux boundary conditions. J. Phys. Oceanogr., 36, 1703-1718.

Belkin, I. M., S. Levitus, J. Antonov, and S.-A. Malmberg, 1998: "Great salinity anomalies" in the North Atlantic. Prog. Oceanogr., 41, 1-68.

Blanke, B., and P. Delecluse, 1993: Variability of the tropical Atlantic Ocean simulated by a general circulation model with two different mixed-layer physics. J. Phys. Oceanogr., 23, 1363-1388.

Bugnion, V., C. Hill, and P. H. Stone, 2006a: An adjoint analysis of the meridional overturning circulation in a hybrid coupled model. J. Climate, 19, 3751-3767.

,$- \ldots$, and $\_, 2006 \mathrm{~b}$ : An adjoint analysis of the meridional overturning circulation in an ocean model. J. Climate, 19, 3732-3750.

Curry, R., and C. Mauritzen, 2005: Dilution of the northern North Atlantic Ocean in recent decades. Science, 308, 1772-1774.

_ B. Dickson, and I. Yashayaev, 2003: A change in the freshwater balance of the Atlantic Ocean over the past four decades. Nature, 426, 826-829.

Delworth, T., S. Manabe, and R. J. Stouffer, 1993: Interdecadal variations of the thermohaline circulation in a coupled ocean-atmosphere model. J. Climate, 6, 1993-2011.

Dijkstra, H. A., and M. Ghil, 2005: Low-frequency variability of the large-scale ocean circulation: A dynamical systems approach. Rev. Geophys., 43, RG3002, doi:10.1029/ 2002RG000122.

Dong, B., and R. T. Sutton, 2005: Mechanism of interdecadal thermohaline circulation variability in a coupled oceanatmosphere GCM. J. Climate, 18, 1117-1135.

Farrell, B. F., and A. M. Moore, 1992: An adjoint method for obtaining the most rapidly growing perturbation to oceanic flows. J. Phys. Oceanogr., 22, 338-349.

_ , and P. J. Ioannou, 1996a: Generalized stability theory. Part I: Autonomous operators. J. Atmos. Sci., 35, 2025-2040.

$\longrightarrow$, and $\longrightarrow$, 1996b: Generalized stability theory. Part II: Nonautonomous operators. J. Atmos. Sci., 53, 2041-2053.

Ganachaud, A., and C. Wunsch, 2000: Improved estimates of global ocean circulation, heat transport and mixing from hydrographic data. Nature, 408, 453-457.

Gent, P. R., and J. C. McWilliams, 1990: Isopycnal mixing in ocean circulation models. J. Phys. Oceanogr., 20, 150-155.

Haak, H., J. Jungclaus, U. Mikolajewicz, and M. Latif, 2003: Formation and propagation of great salinity anomalies. Geophys. Res. Lett., 30, 1473, doi:10.1029/2003GL017065.

Häkkinen, S., 1999: A simulation of thermohaline effects of a great salinity anomaly. J. Climate, 12, 1781-1795.

Huck, T., and G. K. Vallis, 2001: Linear stability analysis of threedimensional thermally-driven ocean circulation: Application to interdecadal oscillations. Tellus, 53A, 526-545.

Josey, S. A., and R. Marsh, 2005: Surface freshwater flux variability and recent freshening of the North Atlantic in the eastern 
subpolar gyre. J. Geophys. Res., 110, C05008, doi:10.1029/ 2004JC002521.

Levitus, S., 1989: Interpentadal variability of temperature and salinity at intermediate depths of the North Atlantic ocean, 1970-1974 versus 1955-1959. J. Geophys. Res., 94, 9679-9685.

Madec, G., and M. Imbard, 1996: A global ocean mesh to overcome the North Pole singularity. Climate Dyn., 12, 381-388.

— Model reference manual. Institut Pierre-Simon Laplace Tech. Rep. 11, 91 pp.

Marotzke, J., 1996: Analysis of thermohaline feedbacks. Decadal Climate Variability: Dynamics and Predictability, D. L. T. Anderson and J. Willebrand, Eds., NATO ASI Series, Vol. 44, Springer, 333-378.

Moore, A. M., and B. F. Farrell, 1993: Rapid perturbation growth on spatially and temporally varying oceanic flows determined using an adjoint method: Application to the Gulf Stream. $J$. Phys. Oceanogr., 23, 1682-1702.

— C. L. Perez, and J. Zavala-Garay, 2002: A non-normal view of the wind-driven ocean circulation. J. Phys. Oceanogr., 32, 2681-2705.

—, J. Vialard, A. T. Weaver, D. L. T. Anderson, and J. R. Johnson, 2003: The role of air-sea interaction in controlling the optimal perturbations of low-frequency tropical coupled ocean-atmosphere modes. J. Climate, 16, 951-968.

Mu, M., and Z. Zhang, 2006: Conditional nonlinear optimal perturbations of a two-dimensional quasigeostrophic model. $J$. Atmos. Sci., 63, 1587-1604.

_ L. Lun, and H. A. Dijkstra, 2004: The sensitivity and stability of the ocean's thermohaline circulation to finite amplitude perturbations. J. Phys. Oceanogr., 34, 2305-2315.

Sévellec, F., M. Ben Jelloul, and T. Huck, 2007: Optimal surface salinity perturbations influencing the thermohaline circulation. J. Phys. Oceanogr., 37, 2789-2808.

Sirkes, Z., and E. Tziperman, 2001: Identifying a damped oscillatory thermohaline mode in a general circulation model using an adjoint model. J. Phys. Oceanogr., 31, 2297-2305.

Stommel, H., 1961: Thermohaline convection with stable regimes flow. Tellus, 13, 224-230.

Sun, L., M. Mu, D.-J. Sun, and X.-Y. Yin, 2005: Passive mechanism of decadal variation of thermohaline circulation. J. Geophys. Res., 110, C07025, doi:10.1029/2005JC002897.

Talley, L. D., J. L. Reid, and P. E. Robbins, 2003: Data-based meridional overturning streamfunctions for the global ocean. J. Climate, 16, 3213-3226.

Tziperman, E., 1997: Inherently unstable climate behaviour due to weak thermohaline ocean circulation. Nature, 386, 592-595.

— citation of thermohaline variability. J. Phys. Oceanogr., 32, $3427-3435$.

Weaver, A. T., J. Vialard, and D. L. T. Anderson, 2003: Threeand four-dimensional variational assimilation with a general circulation model of the tropical Pacific Ocean. Part I: Formulation, internal diagnostics, and consistency checks. Mon. Wea. Rev., 131, 1360-1378.

Zanna, L., and E. Tziperman, 2005: Nonnormal amplification of the thermohaline circulation. J. Phys. Oceanogr., 35, 15931605. 